\title{
Structural Correlates of Mental Health Support Access Among Sexual Minority Youth of Color During COVID-19
}

\author{
Chantelle Roulston, ${ }^{1}$ Sarah McKetta, ${ }^{2}$ Maggi Price, ${ }^{3}$ Kathryn R. Fox ${ }^{4}$ \& Jessica L. Schleider ${ }^{1}$ \\ ${ }^{1}$ Department of Psychology, Stony Brook University \\ ${ }^{2}$ Department of Epidemiology, Columbia University Mailman School of Health \\ ${ }^{3}$ School of Social Work, Boston College \\ ${ }^{4}$ Department of Psychology, University of Denver
}

This manuscript was accepted for publication in Journal of Clinical Child and Adolescent Psychology on January 4, 2022. This version has not been copy-edited and text may change prior to OnlineFirst publication.

\begin{abstract}
Authors' Note
Correspondence concerning this article should be addressed to Dr. Jessica Schleider at jessica.schleider@stonybrook.edu. This work was supported by the NIH Office of the Director [DP5OD028123].
\end{abstract}




\begin{abstract}
Objective: Many youth with mental health needs cannot access treatment, with multiply-marginalized youth, such as sexual minority youth of Color (SMYoC), experiencing both structural and identity-related barriers to care. The COVID-19 pandemic threatens to exacerbate multi-level treatment access barriers facing SMYoC youth nationwide. However, little large-scale research has examined access to mental health care among SMYoC across the United States, either during or prior to the pandemic. Such work is critical to understanding and ameliorating barriers in this domain. Methods: Using data from adolescents who self-identified as SMYoC and who endorsed a desire for mental health support during the COVID-19 pandemic ( $N=470$, ages 13-16, from 43 U.S. states), we examined associations between state-level, structural factors (income inequality; mental healthcare provider shortage; anti-Black racism; homophobia; and the interaction between anti-Black racism and homophobia) and SMYoC mental health treatment access. Results: Multinomial logistic regressions revealed state-level mental healthcare provider shortage as the only significant predictor of SMYoC reporting they never (versus always) accessed mental health support during the COVID-19 pandemic. SMYoC living in areas with both lower homophobia and lower anti-Black racism were more likely to report always (versus sometimes) accessing mental health treatment. Conclusions: Results highlight the critical importance of considering diverse structural factors and applying an intersectional lens when exploring barriers to mental health treatment among multiply-marginalized youth. In locations where provider shortages are less severe, cultural stigma — including anti-Black racism and homophobia — may still pose challenges for SMYoC in need of mental health care.
\end{abstract}

Keywords: Treatment access, sexual minority youth, racial/ethnic minority youth, healthcare disparities, diversity science, youth mental health, intersectionality Preregistration: https://osf.io/vewdg 


\section{Structural Correlates of Mental Health Support Access Among Sexual Minority Youth of Color \\ During COVID-19}

Fewer than $50 \%$ of youth with mental health needs accessed professional treatment prior to the start of the COVID-19 pandemic (Avenevoli et al., 2014). Barriers to accessing services appear especially stark for multiply-marginalized youth, such as sexual minority ${ }^{1}$ youth of Color (i.e. adolescents who selfidentify as a sexual minority - asexual, gay, lesbian, bisexual or otherwise queer in their sexual orientation — and a person of Color; henceforth, SMYoC; Anderson \& Mayes, 2010; Brenner et al., 2018; Macapagal et al., 2016; Wiederhold, 2014). SMYoC routinely report experiencing more difficulty than their White peers in accessing outpatient mental health care (Cook et al., 2016). This inequity may reflect myriad factors, such as cost, convenience and treatment availability, and increased stigma against treatment-seeking in certain racial/ethnic communities (Alegría et al., 2008; Green et al., 2020; Miranda et al., 2015). Likewise, queer youth have lower rates of mental healthcare utilization than heterosexual youth despite the disproportionate adversities they often face, including identity-based stigma—defined as labeling, stereotyping, status loss, or discrimination, wherein one exercises power over others (Link \& Phelan, 2001). Identity-based stigma relates to negative mental health outcomes and can be a major source of stress (Hatzenbuehler et al., 2013; Kates et al., 2017; Macapagal et al., 2016). As SMYoC have two marginalized identities (racial and sexual minority status), they may face compound challenges to accessing mental health treatment when it is needed-particularly during the COVID-19 pandemic, when mental health treatment has simultaneously grown more widespread needed and more difficult to access nationwide (Gruber, Prinstein et al., in press).

To our knowledge, there have been no large-scale evaluations of mental health treatment access among SMYoC during the COVID-19 pandemic. Some prior work (largely conducted pre-pandemic) has explored youths' likelihood of accessing needed mental health treatment, but this work has generally been conducted with majority-White samples and has lacked assessments of youth sexual orientation. To

\footnotetext{
1 The term sexual minority (SM) is used in this research as an umbrella term that broadly captures sexual
} orientations of people who experience societal stigma and oppression (DeBord et al., 2017) 
address this gap, and better understand treatment access among multiply-marginalized youth during the COVID-19 pandemic, we explored rates of accessing needed mental health treatment in a large sample of SMYoC in the United States, as well as system-level factors (anti-Black racism, homophobia, provider shortages, and income inequality) as potential correlates of access rates.

\section{Why Investigating Treatment Access in Multiply-Marginalized Youth Matters}

Racial and sexual minority youth each experience mental health inequities that are at least partially attributable to experiences of interpersonal stigma (Anderson \& Mayes, 2010; Brenner et al., 2018; Wiederhold, 2014). For example, interpersonal racism has been linked to adverse mental health outcomes in youth of Color, including general psychological distress, major depression, anxiety, and lower self esteem (Anderson \& Mayes, 2010; Brenner et al., 2018). Similarly, homophobic victimization and discrimination relate to greater risk for suicidal and nonsuicidal self-injury, anxiety, and depression in sexual minority youth (Wiederhold, 2014).

Youth of Color and sexual minority youth also face unique barriers to accessing mental health support. Intersectionality theory posits that distinct patterns of structural privilege and oppression (e.g., racism, homophobia) result from intersecting social identities at the individual level (e.g., sexual orientation, race; Andersen \& Collins, 2010; Bowleg, 2012; Crenshaw, 1989). Research applying intersectional frameworks has suggested intersecting minority identities (e.g., race, ethnicity, sexual orientation) may adversely affect youth experiences in healthcare for example experiencing decreased access to care and receiving subjectively less adequate care (Andersen \& Collins, 2010; Bowleg, 2012; Crenshaw, 1989; Macapagal et al., 2016; Price, 2018).

Identifying as both a racial and sexual minority may present a greater risk for mental health issues and barriers to care due to compounding effects of oppression and discrimination on multiplymarginalized identities (Macapagal et al., 2016; Price, 2018; Price et al., 2019; Whitfield et al., 2014). Not only are SMYoC at an increased risk for mental health problems relative to their White and heterosexual peers (Brenner et al., 2018; Wiederhold, 2014), but they may also face identity-related structural barriers that could undermine their access support for those problems. Accordingly, it is imperative to examine 
multiple structural factors that may shape accessing mental health support for youth in this multiply marginalized group.

\section{Why Structural Barriers Matter for Youth Mental Health Treatment Access}

Many barriers to accessing mental health supports have been identified in the broader treatment literature. Prior research has centered individual-level predictors (e.g., symptom severity, internalized stigma) and person-level barriers to accessing mental health care (e.g., familial support; Anderson \& Mayes, 2010; Burgess et al., 2007; Cummings et al., 2013). However, structural obstacles embedded within an environment may also undermine youths' access to mental health support. One such category of barriers, structural stigma, has been identified as a robust mechanism underlying poor mental health related to sexual orientation among youth and can shape the lives of stigmatized individuals (Hatzenbuehler, 2016; Link \& Phelan, 2001). Structural stigma reflected (as examples) by shortages of healthcare providers with expertise in treating sexual minority youth of Color, can exacerbate health disparities, contribute to various adverse health outcomes, and may also undermine mental health treatment access among youth (Hatzenbuehler, 2016; Hatzenbuehler, et al., 2013). Although many structural factors hold potential to shape access to mental health support among SMYoC, the present study examined four such barriers that previous research suggests may be relevant: structural homophobia and anti-Black racism, shortages in mental health providers, and income inequality.

One way in which structural stigma may manifest is via community beliefs and attitudes (e.g., cultural homophobia and cultural racism $^{2}$, including explicit, negative beliefs and perceptions about racial and sexual minority groups). These beliefs have been shown to directly influence health and mental health (Hatzenbuehler et al., 2017; Hatzenbuehler \& Pachankis, 2016; Link \& Phelan, 2001) influencing if, how, and when people choose to access treatment (Cauce et al., 2002). As such, these attitudes may impact access to care among SMYoC, although this prospect has yet to be evaluated formally.

\footnotetext{
${ }^{2}$ Cultural racism: the instillation of the ideology of inferiority in the values, language, imagery, symbols, and unstated assumptions of the larger society (Williams et al., 2019)
} 
Mental health provider shortage areas (MHPSAs) represent a second structural barrier of potential importance to treatment access among SMYoC. There is currently a national shortage of professional mental health providers, especially those that specialize in working with youth. The Health Resources and Services Administration categorizes four fifths of U.S. counties as Mental Health Professional Shortage Areas (Cummings et al., 2013; Health Resources and Services Administration), meaning that there is a greater need for mental health services than there are professional providers to fulfill this need in these places. Youths who live in MHPSAs thus face a clear mental health treatment access barrier: even if they want and seek out support, a local shortage of trained providers may prevent them from receiving it.

A third structural barrier to treatment access among SMYoC is low income, which can preclude access to mental health care. Living in a low-income home has been linked to greater risk for mental health problems than living in a middle- or high-income home among youth, and youth living at or below the federal poverty level (an income of $\leq \$ 23624$ for a family of 4 with 2 children) are least likely to be connected with high-quality mental health care (Hodgkinson et al., 2017).

\section{Present Study}

The present study examined structural predictors of mental health support access during the COVID-19 pandemic among SMYoC with elevated depressive symptoms in the U.S. We explored how frequently SMYoC reported accessing mental health support, when desired and whether SMYoC living in states characterized by varying levels of anti-Black racism, homophobia, mental health care provider shortages, or income inequality reported differential access to mental health support. More specifically, we explored three primary questions:

(1) How frequently are SMYoC adolescents 13-16 able to access mental health support when they need it during the COVID-19 pandemic?

(2) Do SMYoC adolescents living in states characterized by higher anti-Black racism and higher homophobia report less access to mental health support, relative to those living in states with lower levels of anti-Black racism and lower levels of homophobia? 
(3) Do state-levels of homophobia and anti-Black racism relate to adolescents' perceived access to mental health support over and above local availability of mental healthcare providers and state-level income inequality?

\section{Method}

\section{Participants}

This pre-registered, secondary analysis study (https://osf.io/vewdg) used data from a subset of adolescents who completed the baseline (pre-intervention) survey battery within a web-based randomized controlled trial (NCT04634903). Specifically, data from adolescents who (1) self-identified as sexual minority youth of Color (SMYoC), and (2) who endorsed any interest in accessing mental health support since the COVID-19 pandemic began in February 2020 were included in this study $(N=470$, ages $13-16)$. Inclusion criteria for participation in the larger trial were (1) being between 13 and 16 years old (inclusive) at the time of enrollment; (2) comfort reading and writing in English; (3) internet and computer, laptop, or smartphone access; and (4) endorsement of elevated depressive symptoms, per an eligibility screener score of $>=2$ on the Patient Health Questionnaire -2 item version (PHQ-2).

Adolescents who met criteria for the present study were $88.94 \%$ female (sex assigned at birth) and $6.38 \%$ American Indian, 22.34\% Asian, 20.00\% Black, 4.47\% Native Hawaiian, $56.81 \%$ Latinx, and 16.17\% White (participants could endorse multiple racial and ethnic categories). Regarding sexual orientation, $5.53 \%$ identified as asexual, $35.96 \%$ as bisexual, $11.70 \%$ as gay/lesbian/homosexual, $16.81 \%$ as pansexual, $13.83 \%$ as unsure or questioning, $6.38 \%$ as queer, $3.62 \%$ as "other," and $6.17 \%$ reported not using any label for their sexual orientation. Regarding geographic location, adolescents in this study lived in 43 different U.S. states, with the largest groups living in California (19.82\%), Texas (10.91\%), Florida (8.91\%), New York (6.46\%), Colorado (3.79\%), and Georgia (3.56\%). States not represented in this sample were Alaska, Arkansas, North Dakota, South Dakota, Rhode Island, West Virginia, and Vermont.

\section{Procedure}


All study procedures were approved by the University IRB prior to participant enrollment, and all adolescents provided online assent prior to beginning the study (see Schleider et al., 2021, for original study procedures). Participants learned about the study through Instagram advertisements (total costs $<\$ 2,000)$, which followed established ethics guidelines for passive, social media-based recruitment (Gelinas et al., 2017). Posts included invitations to determine eligibility for a confidential, online psychology study, for which participants could earn up to \$20 USD in gift cards across a 3-month period. Instagram posts linked to a Qualtrics survey, the first page of which included details about the project and an invitation to complete an eligibility screener. Participants could complete the screener and the study from any internet-equipped device. In order to maintain anonymity and minimize access barriers (e.g., discomfort disclosing psychological distress, as parents are often unaware of their adolescents' depressive symptoms, including suicidal and morbid ideation, in up to $80 \%$ of cases (e.g., Baumgartner et al., 2020; Eskin, 2003; Simone \& Hamza, 2020), parent permission was not required to participate in this study (waived by the University IRB).

After clicking on the post, adolescents were directed to an informational study webpage inviting them to complete an online eligibility screener. Eligible adolescents then reviewed an online assent form inviting them to participate. Adolescents could initiate the study at any time and location, using any internet-equipped device (smartphone, laptop, tablet). After starting the study, youth completed a battery of self-report questionnaires; the full measurement battery is listed in the original trial registration (NCT04634903), and study-relevant items are detailed below. All recruitment and data collection took place between November and December of 2020.

\section{Measures}

Demographics. Participants self-reported their age (13 to 16, in years), sex assigned at birth, sexual orientation, race/ethnicity, and present home zip code. Participants were offered multiple-choice options as well as a write-in option labeled "other (please specify)" for questions regarding sexual orientation and race/ethnicity. 
Access to Mental Health Support During the COVID-19 Pandemic. The outcome of interest was the level of self-reported access to professional mental health treatment or support, when a participant wanted it, during the COVID-19 pandemic. To define "mental health support or treatment," participants were presented with the following examples: "talking with a school counselor or out-ofschool therapist about your stress or problems, or getting medications for mental health problems from a medical doctor." Participants' responses to this item were operationalized as a 3-level categorical outcome variable: "Since the pandemic started, I have ALWAYS been able to get mental health support when I wanted it," "Since the pandemic started, I have SOMETIMES been able to get mental health support when I wanted it," and "Since the pandemic started, I have NEVER been able to get mental health support when I wanted it." Given the specificity of our outcome of interest (i.e., mental health treatment access during the pandemic among adolescents who wanted it), this item was developed specifically for the present study. Of note, due to pre-registered inclusion criteria, adolescents who selected a fourth response option to this item-“Does Not Apply: I have not wanted mental health treatment since the pandemic started"—-were excluded from present analyses.

Structural predictors of adolescent treatment access. Structural predictors in this study were state-level anti-Black racism, state-level homophobia, mental health care provider shortage severity, and income inequality. All predictors were computed at the state level; every state had a single score for each predictor, and values for each predictor were matched to participants per their self-reported home zip codes (see Online Supplement).

State-level anti-Black racism and homophobia were quantified using factor analyses on publiclyavailable data drawn from Project Implicit (https://osf.io/y9hiq/), using explicit questions from two modules (Race Implicit Association Test and Sexuality Implicit Association Test modules) from the years 2015-2019. Briefly, individual responses from Likert-style indicators related to anti-Black racism $(N=37$ candidate indicators) and homophobia ( $N=7$ candidate indicators) were scored such that higher values reflected higher animus, averaged to the state-level, and mean-standardized. Anti-Black racism and homophobia were modeled separately. Scree plots and parallel analyses were run to determine 
dimensionality of the underlying factor(s), and exploratory factor analysis was used to determine item loadings. Items that loaded at $<0.6$ were removed from consideration, and factor analyses were re-run with the retained items and model-based factor scores were assigned to each state. For anti-Black racism, a unidimensional solution with 21 items explaining $63 \%$ of the variance was retained. For homophobia, a unidimensional solution with 5 items explaining $87 \%$ of the variance was retained. Model-based factor scores were output from each model, assigning a score for anti-Black racism and homophobia to each state. Retained indicators for each model and their factor loadings are shown in Supplemental Table 1, and descriptive statistics for all state-level predictor variables are noted in Supplemental Table 2.

The Gini coefficient is a measure of income inequality, ranging from 0 (perfect equality) to 1 (perfect inequality), and was obtained for each state from the 2019 ACS. Information regarding Mental Health Provider Shortage Areas (HPSAs; Wang \& Luo, 2005) for mental health care was drawn from the Health Resources and Services Administration (HRSA), which computes HPSA scores (ranging from 0 to 25) across all counties in the United States. It is computed as an index score of 7 different indicators: the Population-to-Provider Ratio (up to 7 points), the percent of population below $100 \%$ Federal Poverty Level (FPL) (up to 5 points), the elderly ratio (percent of people over age 65) (up to 3 points), the youth ratio (percent of people under age 18) (up to 3 points), alcohol abuse prevalence (1 point), substance abuse prevalence (1 point), and travel time to Nearest Source of Care (NSC) outside the HPSA designation area (up to 5 points). These scores were obtained as an average for the year 2019 from a public, online database (https://data.hrsa.gov/tools/shortage-area/hpsa-find).

Statistical analysis. Per our pre-registered analytic plan, associations between state-level, structural predictors (income inequality; mental health care provider shortage; anti-Black racism; homophobia; and the interaction between anti-Black racism and homophobia) and SMYoC mental health treatment access were measured using multinomial logistic regression. The reference category for this test was "always" accessing mental health treatment, when needed; thus, results yielded odds ratios (OR) for the effect of each structural predictor on the likelihood of SMYoC sometimes or never accessing mental health treatment, relative to the odds of their always accessing that treatment. Sequential, within-family 
Holm-Bonferroni corrections were applied, and both unadjusted and adjusted $p$-values are reported below. Regarding missing data, we included all participants for whom key data were reported (zip code; sexual orientation; race/ethnicity; mental health treatment access during the COVID-19 pandemic). This process resulted in the exclusion of data from 21 of $470 \mathrm{SMYoC}$ from our final model, for whom zip codes were unreported. Data from all other SMYoC were included in analyses. Alpha was set at $p<.05$ for our multinomial logistic regression model; reported $p$-values reflect Holm-Bonferonni corrected values.

\section{Results}

Descriptive statistics. Table 1 reports the total number of SMYoC who were always, sometimes, and never able to access mental health treatment during the pandemic, among those who endorsed wanting treatment, by sexual orientation and racial/ethnic identity. Overall, $43.4 \%$ of participants were never able to access support; $40.4 \%$ sometimes accessed support; and $16.2 \%$ always accessed support. Within certain identity groups, access rates were numerically poorer; for instance, only $12.43 \%$ of bisexual adolescents, $13.92 \%$ of pansexual adolescents, $6.90 \%$ of American Indian adolescents, and 9.5\% of Native Hawaiian adolescents reported "always" accessing mental health treatment when they wanted it, since the pandemic began. Due to low numbers by cell that would render test results difficult to interpret, we did not examine these numeric discrepancies statistically.

We also examined correlations among pre-registered model predictors. As expected, mean statelevel mental health care provider shortages were correlated positively with state-level income inequality $(r=0.31, p<.001)$, and state-level anti-Black racism and state-level homophobia were strongly correlated with each other $(r=0.70, p<.001)$. Anti-Black racism was not significantly associated with state-level mental health care provider shortages, and greater state-level income inequality (often reflecting the presence of densely-populated, liberal-leaning cities within the state, where income inequalities tend to be extreme) was associated with lower state-level homophobia.

Did state-level provider shortage, income inequality, anti-Black racism, homophobia, or their interaction predict mental health treatment among SMYoC? Full results of the multinomial logistic regression model are presented in Table 2, including adjusted and unadjusted $p$-values. During 
the COVID-19 pandemic, SMYoC living in states with more severe mental health care provider shortages were more likely to never (versus always; $\mathrm{OR}=1.52,95 \% \mathrm{CI}[1.19,1.94]$, adjusted $p=.001$ ) or sometimes (versus always; $\mathrm{OR}=1.34,95 \% \mathrm{CI}[1.07,1.68]$, adjusted $p=0.01$ ) access desired mental health treatment. Additionally, a significant racism*homophobia interaction effect emerged, such that SMYoC living in areas with lower homophobia and lower anti-Black racism were more likely to report always (versus sometimes) accessing mental health treatment ( $\mathrm{OR}=0.61,95 \%$ CI $[0.40,0.93]$, adjusted $p$ $=.04)$. However, state-level provider shortage - not income inequality, racism, homophobia, or their interaction — was the only significant predictor of SMYoC reporting they never (versus always) accessed mental health support during the COVID-19 pandemic.

We computed and plotted estimated marginal means to gauge the nature of the significant racism*homophobia interaction effect for the sometimes versus always comparison, reflecting the probability of SMYoC always, sometimes, and never accessing mental health treatment in states characterized by low (-1SD), average, and high (+1SD) levels of structural anti-Black racism and homophobia, respectively (see Figure 1 and Supplemental Table 3). The observed interaction effect appeared to be driven by variations in youth "always" accessing treatment, as a function of state-level homophobia and anti-Black racism. Specifically, SMYoC with the greatest odds of "always" accessing desired mental health treatment lived in states with low levels of both anti-Black racism and homophobia (probability $=0.17,95 \%$ CI $[.11, .23])$. Probabilities of "always" accessing mental health treatment were numerically lower in states with average or high levels of either anti-Black racism or homophobia, suggesting that elevations in either form of stigma are linked with decreased access to care among SMYoC.

\section{Discussion}

During the COVID-19 pandemic, across 43 U.S. states, the vast majority (83.8\%) of SMYoC were either never or only sometimes able to access mental health support when they wanted it. SMYoC living in states with more severe mental health care provider shortages were significantly more likely to never or sometimes (versus always) access the mental health treatment that they wanted. SMYoC living in 
areas with lower homophobia and lower anti-Black racism were significantly more likely to always (versus sometimes) access mental health treatment. Among anti-Black racism, homophobia, mental health provider shortages and income inequality, state-level mental health provider shortage was the most consistent predictor of SMYoC never (versus always) accessing support. SMYoC with the greatest odds of "always" accessing desired mental health treatment lived in states with lower levels of both anti-Black racism and homophobia. Likewise, youths" odds of "always" accessing support was lower in states with higher levels of either anti-Black racism or homophobia.

In this sample, the majority (84\%) of SMYoC who desired mental health treatment during COVID-19 were not receiving it. The need for mental health treatment among adolescents has increased significantly during the COVID-19 pandemic, as evidenced by the global rise of anxiety and depression in young people (Racine et. al, 2021), yet the majority of SMYoC in this sample reported not accessing desired help. Notably, the need for mental health care may be particularly high for SMYoC, relative to their non-minoritized peers, given identity-related stressors that have occurred throughout the pandemic (e.g., increases in attention to racial disparities and recently passed anti-trans laws in the United States). Given these realities, it is critical important to target this demographic, in particular, when designing and disseminating mental health supports and resources, during and beyond the pandemic. Additionally, results suggest that certain structural barriers were indeed related to mental health treatment access for SMYoC, which may inform next-steps for disseminating care more successfully. In this sample, local shortages of trained mental health providers corresponded to fewer youth accessing desired support. This finding, in conjunction with the fact that the Health Resources and Services Administration categorizes four-fifths of US counties as mental health provider shortage areas, reinforces the importance of structural factors - and geospatial factors specifically —in understanding and ameliorating treatment access barriers facing SMYoC (Health Resources and Services Administration; Cummings, Wen, \& Druss, 2013). Facilitating access to mental health care by prioritizing these potentially high impact geographic locations when disseminating treatments may be beneficial for SMYoC living in high areas with greater provider shortage severity. Specifically, training providers in these areas to provide culturally-competent care to 
LGBQ and BIPOC youth may make the most impactful difference for SMYoC. Furthermore, expanding availability of online resources, evidence-based online interventions, and remote access to mental health providers specifically for SMYoC may help to close the accessibility gap. If there is insufficient support for SMYoC in particular states, digital supports tailored to this group may expand options and address their needs specifically. Digital mental health supports in particular may help to fill this gap, especially during the COVID-19 pandemic, when many in-person mental health services have been rendered unavailable. In fact, some freely-available, online mental health are equally acceptable and useful for LGBTQ+ adolescents experiencing depression and similar difficulties (McDanal et al., 2021). Simultaneously, future research might examine how to ameliorate provider shortages in states with few youth-focused mental health providers. Currently, some structural solutions exist, including an initiative by the Centers for Medicare and Medicaid offering monetary incentives (e..g, 10\% bonus payments) for mental health clinicians who practice in MHPSAs. However, there remains a dearth of mental health providers in many areas, indicating needs for new solutions. Structural changes, such as loan forgiveness for mental health clinicians working in MHPSAs, may be especially useful, as similar programs already exist for physicians.

A significant racism*homophobia interaction effect emerged, such that SMYoC living in areas with lower homophobia and lower anti-Black racism were more likely to report always (versus sometimes) accessing mental health treatment, and youth in states with average or high levels of antiBlack racism or homophobia were less likely to "always" access mental health support. These results suggest that levels of anti-Black racism and homophobia together may relate to SMYoC access to mental health support. It may be important for policy makers and researchers to be thoughtful and intentional about efforts to disseminate mental health treatments, remaining mindful of where and to whom those treatments are successfully disseminated. Prioritizing dissemination of support directly to SMYoC in areas with higher levels of anti-Black racism and homophobia-for example, by soliciting SMYoC input on how they would prefer to access mental health supports - may strengthen their likelihood of receiving desired care. It may be particularly important to prioritize SMYoC who are living in areas characterized 
by higher levels of anti-Black racism and homophobia, as adverse experiences related to homophobia and racism may worsen as a result of increased isolation caused by statewide, COVID-19 lockdowns (Salerno, Williams, Gattamorta, 2020). Simultaneously, it remains critical to consider the cultural acceptability of treatments disseminated to SMYoC_-including assurances that providers are properly trained to serve this unique population, and that services are affordable and affirming to SMYoC. It is also notable that statelevel anti-Black racism and state-level homophobia were strongly, but not perfectly, correlated with one another $(r=0.70, p<.001)$. Results indicate that the presence of either homophobia or racism is associated with lower access to care-and SMYoC are most likely to consistently access care in states where both are low. The findings of this study reinforce the importance of considering structural stigma through an intersectional lens, along with the need to address multiple types of stigma to enable treatment access in diverse youth populations.

Study results also beg the question of why provider shortages were more consistently linked to treatment access relative to cultural attitudes in a sample of multiply-marginalized youth. It is possible that, in places where there are very few mental health providers, access to care may be so lacking that the impacts of racism and homophobia are difficult to detect. These results do not imply that racism and homophobia do not matter; indeed, these cultural attitudes may become more apparent as access to providers increases, at which point it may be easier to detect factors beyond provider shortages in hindering access to care. When youth mental healthcare providers are exceptionally scarce, there is little opportunity for racism, homophobia, or any system-level factor to further undermine treatment access.

Several study limitations warrant consideration. First, measurement specificity may have impacted results. This study examines anti-Black racism specifically which does not apply to everyone equally, as there are various forms of racism which may have differently affected study results, especially as we worked with a racially diverse sample of whom $20 \%$ identified as Black. Additionally, we examined state-level indicators of anti-Black racism and homophobia, however state level differences can mask meaningful heterogeneity at the city or county level, or may be an insufficient proxy for more proximate experiences. Second, collinearity among predictor variables (such as mental health provider 
shortages and income inequality) pose a potential limitation, as these factors are impossible to fully disentangle. Collinearity is a feature of work in this area that makes it slightly more challenging. Finally, the low representation of the "never" accessing desired mental health treatment category limits our ability to detect differences between "never" and other categories. Moving ahead, future studies may be strengthened by examining various forms of racism, complementing the Sexuality Implicit Association Test module with additional attitudinal measures of sexual minority individuals, and examining varying levels of indicators of racism and homophobia.

This study used data from a large racially and sexual orientation diverse sample with individuals from almost every U.S. state. Results highlight the critical importance of considering diverse structural factors, and applying an intersectional lens to understanding stigma when exploring barriers to mental health treatment among multiply-marginalized youth. The availability of mental health care providers in the area appears centrally important to SMYoC access to mental health support during the COVID-19 pandemic. However, in locations where provider shortages are less severe, structural stigma—including anti-Black racism and homophobia-may still pose challenges for SMYoC in need of mental health care. 


\section{References}

Alegría, M., Chatterji, P., Wells, K., Cao, Z., Chen, C., Takeuchi, D., Jackson, J., \& Meng, X. (2008). Disparity in Depression Treatment Among Racial and Ethnic Minority Populations in the United States. Psychiatric Services, 59(11), 1264-1272. https://doi:10.1176/ps.2008.59.11.1264

Anderson, E. R., \& Mayes, L. C. (2010). Race/ethnicity and internalizing disorders in youth: A review. Clinical Psychology Review. 30(3), 338-348 https://doi.org/10.1016/j.cpr.2009.12.008

Avenevoli, S., Swendsen, J., He, J. P., Burstein, M., \& Merikangas, K. R. Major depression in the National Comorbidity Survey-Adolescent Supplement: prevalence, correlates, and treatment. Journal of the American Academy of Child \& Adolescent Psychiatry 54, 37-44 (2015). https://doi.org/10.1016/j.jaac.2014.10.010

Beavers, A. S., Lounsbury, J. W., Richards, J. K., Huck, S. W., Skolits, G. J., \& Esquivel, S. L. (2013). Practical considerations for using exploratory factor analysis in educational research. Practical Assessment, Research, and Evaluation, 18(1), 6.

Bowleg, L. (2012). The problem with the phrase women and minorities: Intersectionality-An important theoretical frame- work for public health. American Journal of Public Health, 102(7), 12671273. https://doi:10.2105/ajph.2012.300750

Brenner, A. D., Wang, Y., Shen, Y., Boyle, A. E., Polk, R., \& Cheng, Y. (2018) Racial/Ethnic Discrimination and Well-Being During Adolescence: A Meta-Analytic Review. American Psychologist. https://doi.org/10.1037/amp0000204.supp

Burgess D., Lee R., Tran A., \& Van Ryn M. (2007) Effects of Perceived Discrimination on Mental Health and Mental Health Services Utilization Among Gay, Lesbian, Bisexual and Transgender Persons, Journal of LGBT Health Research, 3:4, 1-14, https://doi.org/10.1080/15574090802226626

Cauce, A. M; Domenech-Rodríguez, M., Paradise, M. Matthew; Cochran, B.N.;Shea, J.M., Srebnik, D., \& Baydar, N. (2002). Cultural and contextual influences in mental health help seeking: A focus 
on ethnic minority youth. Journal of Consulting and Clinical Psychology, 70(1), 44-55. https://doi:10.1037/0022-006x.70.1.44

Cook, B., Trinh, N. H., Li, Z., Hou, S. S., \& Progovac, A. M. (2016). Trends in Racial-Ethnic Disparities in Access to Mental Health Care, 2004-2012. Psychiatric Services. https://doi.org/10.1176/appi.ps.201500453

Crenshaw, K. (1989). Demarginalizing the intersection of race and sex: A black feminist critique of antidiscrimination doctrine, feminist theoryand antiracist politics. University of Chicago Legal Forum, 1989, 139-167.

Cummings, J. R., Wen, H., \& Druss, B. G. (2013). Improving Access to Mental Health Services for Youth in the United States. JAMA, 309(6), 553. https://doi.org/10.1001/jama.2013.437

DeBord, K. A., Fischer, A .R., Bieschke, K. J., \& Perez, R. M. (Eds.). (2017). Handbook of sexual orientation and gender diversity in counseling and psychotherapy. Washington, DC: American Psychological Association.

Green, J. G., McLaughlin, K. A., Fillbrunn, M., Fukuda, M., Jackson, J. S., Kessler, R. C., Sadikova, E., Sampson, N. A., Vilsaint, C., Williams, D. R., Cruz-Gonzalez, M. \& Alegría, M. (2020). Barriers to Mental Health Service Use and Predictors of Treatment Drop Out: Racial/Ethnic Variation in a Population-Based Study. Adm Policy Mental Health 47, 606-616. https://doi.org/10.1007/s10488-020-01021-6

Gruber, J., Prinstein, M. J., Clark, L. A., Rottenberg, J., Abramowitz, J. S., Albano, A. M., Aldao, A., Borelli, J. L., Chung, T., Davila, J., Forbes, E. E., Gee, D. G., Hall, G. C. N., Hallion, L. S., Hinshaw, S. P., Hofmann, S. G., Hollon, S. D., Joormann, J., Kazdin, A. E., ... \& Weinstock, L. M. (2020) Mental Health and Clinical Psychological Science in the Time of COVID-19: Challenges, Opportunities, and a Call to Action. American Psychologist. https://doi.org/10.1037/amp0000707.

Hatzenbuehler, M. L. (2016). Advancing Research on Structural Stigma and Sexual Orientation Disparities in Mental Health Among Youth. Journal of Clinical Child \& Adolescent Psychology, 
1-13. https://doi.org/10.1080/15374416.2016.1247360 Hatzenbuehler, M. L. (2016). Structural stigma: Research evidence and implications for psychological science. American Psychologist, 71(8), 742-751. https://doi.org/10.1037/amp0000068

Hatzenbuehler, M. L., Flores, A. R., \& Gates, G. J. (2017). Social Attitudes Regarding Same-Sex Marriage and LGBT Health Disparities: Results from a National Probability Sample. Journal of Social Issues, 73(3), 508-528. https://doi.org/10.1111/josi.12229

Hatzenbuehler, M. L., Phelan, J. C., \& Link, B. G. (2013). Stigma as a Fundamental Cause of Population Health Inequalities. American Journal of Public Health, 103(5), 813-821. https://doi.org/10.2105/ajph.2012.301069

Hatzenbuehler, M. L., \& Pachankis, J. E. (2016). Stigma and Minority Stress as Social Determinants of Health Among Lesbian, Gay, Bisexual, and Transgender Youth. Pediatric Clinics of North America. https://doi.org/10.1016/j.pcl.2016.07.003

Homan, P. (2019). Structural Sexism and Health in the United States: A New Perspective on Health Inequality and the Gender System. American Sociological Review, 84(3), 486-516. doi:10.1177/0003122419848723

Kates, J., Ranji, U., Beamesderfer, A., Salganicoff, A., \& Dawson, L. (2015) Health and Access to Care and Coverage for Lesbian, Gay, Bisexual, and Transgender Individuals in the U.S. Kaiser Family Foundation.

Kline, P. (2014). An Easy Guide to Factor Analysis. http://www.vlebooks.com/vleweb/product/openreader?id=none\&isbn=9781315788135

Link, B. G., \& Phelan, J. C. (2001). Conceptualizing stigma. Annual Review of Sociology, (27)363-385. https://doi.org/10.1146/annurev.soc.27.1.363

Macapagal, K., Bhatia, R., \& Greene, G. J. (2016) Differences in Healthcare Access, Use, and Experiences Within a Community Sample of Racially Diverse Lesbian, Gay, Bisexual, 
Transgender, and Questioning Emerging Adults. LGBT Health (6) 434-442. https://doi.org/10.1089/lgbt.2015.0124.

McDanal, R., Rubin, A., Fox, K., \& Schleider, J. L. (2021). Associations of LGBTQ+ identities with acceptability and efficacy of online single-session youth mental health interventions. Behavior Therapy. https://doi.org/10.1016/j.beth.2021.10.004

Miranda, R., Soffer, A., Polanco-Roman, L., Wheeler, A., \& Moore, A. (2015). Mental Health Treatment Barriers Among Racial/Ethnic Minority Versus White Young Adults 6 Months After Intake at a College Counseling Center. Journal of American College Health, 63(5), 291-298. https://doi.org/10.1080/07448481.2015.1015024

Pachankis, J. E., Hatzenbuehler, M. L., Berg, R. C., Fernández-Dávila, P., Mirandola, M., Marcus, U., ... \& Schmidt, A. J. (2017). Anti-LGBT and anti-immigrant structural stigma: an intersectional analysis of sexual minority men's HIV risk when migrating to or within Europe. Journal of acquired immune deficiency syndromes (1999), 76(4), 356.

Pachankis, J. E., Hatzenbuehler, M. L., Hickson, F., Weatherburn, P., Berg, R. C., Marcus, U., \&

Schmidt, A. J. (2015). Hidden from health: structural stigma, sexual orientation concealment, and HIV across 38 countries in the European MSM Internet Survey. AIDS (London, England), 29(10), 1239.

Price, M. (2018). The Effects of Identity-Based Victimization on Youth: An Intersectional Examination of Mental health, Academic Achievement, and the Impact of Teacher-Student Relationships. http://hdl.handle.net/2345/bc-ir:107608.

Price, M., Hill, N. E., Polk, W., Liang, B. (2019). The intersectionality of identity-based victimization in Adolescence: A person-centered examination of mental health and academic achievement in a U.S. high school. Journal of Adolescence. https://doi.org/10.1016/j.adolescence.2019.09.002

Price, M. A., Weisz, J. R., McKetta, S., Lattanner, M. R., Reid, A. E., \& Hatzenbeuhler, M. L. (2021) Meta-analysis: Are Psychotherapies Less Effective for Black Youth in Communities With Higher Levels of Anti-Black Racism? Journal of the American Academy of Child \& Adolescent Psychiatry https://doi.org/10.1016/j.jaac.2021.07.808 
Racine, N., McArthur, B. A., Cooke, J. E., Eirich, R., Zhu, J., \& Madigan, S. (2021). Global prevalence of depressive and anxiety symptoms in children and adolescents during covid-19: A metaanalysis. JAMA Pediatrics, 175(11), 1142-1150. https://doi.org/10.1001/jamapediatrics.2021.2482

Reid, A. E., Dovidio, J. F., Ballester, E., \& Johnson, B. T. (2014). HIV prevention interventions to reduce sexual risk for African Americans: The influence of community-level stigma and psychological processes. Social science \& medicine, 103, 118-125.

Salerno, J. P., Williams, N. D., \& Gattamorta, K. A. (2020). LGBTQ populations:

Psychologically vulnerable communities in the COVID-19 pandemic. Psychological Trauma: Theory, Research, Practice, and Policy, 12(S1), S239 https://doi.org/10.1037/tra0000837

Schleider, J. L., Mullarkey, M. C., Fox, K., Dobias, M., Shroff, A., Hart, E., \& Roulston, C. A. (2021). Single-Session Interventions for Adolescent Depression in the Context of COVID-19: A Nationwide Randomized-Controlled Trial. Nature Human Behaviour. https://doi.org/10.1038/s41562-021-01235-0

Tumin, D., Menegay, M., Shrider, E. A., Nau, M., \& Tumin, R. (2018) Local Income Inequality, Individual Socioeconomic Status, and Unmet Healthcare Needs in Ohio, USA Health Equity. 37(44) http://doi.org/10.1089/heq.2017.0058

US Department of Health and Human Services. 2011-2012 Area Resource File (ARF). Health Resources and Services Administration website. https://arf.hrsa.gov

Whitfield, D. L., Walls, N.E., Langenderfer-Magruder, L., \& Clark, B. (2014). Queer Is the New Black? Not So Much: Racial Disparities in Anti-LGBTQ Discrimination. Journal of Gay \& Lesbian Social Services, 26(4), 426-440. doi.org/10.1080/10538720.2014.955556

Wiederhold, B. K. (2014). Cyberbullying and LGBTQ Youth: A Deadly Combination. Cyberpsychology, Behavior, and Social Networking, 17(9), 569-570. https://doi.org/10.1089/cyber.2014.1521 
Williams, D. R., Lawrence, J. A., \& Davis, B. A. (2019). Racism and Health: Evidence and Needed Research. Annual Review of Public Health, 40(1) https://doi.org/10.1146/annurev-publhealth040218-043750

OED Online, Oxford University Press (2021) www.oed.com/view/Entry/156236. Accessed 10 March 2021 
Table 1. Frequencies of SMYoC Mental Health Treatment Access During the Pandemic by Sexual Orientation and Race/Ethnicity

Sexual Orientation (participants could select one category each)

\begin{tabular}{|c|c|c|c|c|c|c|c|c|}
\hline $\begin{array}{l}\text { Able to access } \\
\text { mental health } \\
\text { treatment when } \\
\text { wanted? }\end{array}$ & Asexual & Bisexual & $\begin{array}{l}\text { Gay/ } \\
\text { Lesbian/ } \\
\text { Homo- } \\
\text { sexual }\end{array}$ & $\begin{array}{c}\text { Do } \\
\text { not } \\
\text { use a } \\
\text { label }\end{array}$ & Other & Queer & Pansexual & $\begin{array}{c}\text { Unsure/ } \\
\text { Questioning }\end{array}$ \\
\hline $\begin{array}{l}\text { ALWAYS able } \\
\text { to access } \\
\text { treatment }\end{array}$ & 4 & 21 & 12 & 4 & 4 & 6 & 11 & 14 \\
\hline $\begin{array}{l}\text { SOMETIMES } \\
\text { able to access } \\
\text { treatment }\end{array}$ & 16 & 76 & 22 & 9 & 6 & 16 & 33 & 26 \\
\hline \multirow{3}{*}{$\begin{array}{l}\text { NEVER able to } \\
\text { access treatment }\end{array}$} & 6 & 72 & 21 & 16 & 7 & 8 & 35 & 25 \\
\hline & \multicolumn{8}{|c|}{ Race/Ethnicity (participants could select $\geq 1$ categories each) } \\
\hline & Black & $\begin{array}{l}\text { Latinx/ } \\
\text { Hispanic }\end{array}$ & Asian & Other & \multicolumn{2}{|c|}{$\begin{array}{l}\text { American } \\
\text { Indian }\end{array}$} & $\begin{array}{c}\text { Native } \\
\text { Hawaiian }\end{array}$ & White \\
\hline $\begin{array}{l}\text { ALWAYS able } \\
\text { to access } \\
\text { treatment }\end{array}$ & 18 & 41 & 12 & 1 & \multicolumn{2}{|c|}{2} & 2 & 13 \\
\hline $\begin{array}{l}\text { SOMETIMES } \\
\text { able to access } \\
\text { treatment }\end{array}$ & 37 & 121 & 44 & 10 & \multicolumn{2}{|c|}{16} & 12 & 37 \\
\hline $\begin{array}{l}\text { NEVER able to } \\
\text { access treatment }\end{array}$ & 39 & 105 & 49 & 9 & \multicolumn{2}{|c|}{12} & 7 & 26 \\
\hline
\end{tabular}


Table 2. Multinomial Logistic Regression Testing Association of State-Level Variables to Mental Health Treatment Access During the Pandemic among Sexual Minority Youth of Color

\begin{tabular}{|c|c|c|c|c|c|c|c|c|c|}
\hline \multirow[b]{2}{*}{$\begin{array}{c}\text { Mental Healthcare } \\
\text { Access }\end{array}$} & \multirow[b]{2}{*}{$\begin{array}{l}\text { State-Level } \\
\text { Predictor }\end{array}$} & \multirow[b]{2}{*}{ Est. } & \multirow[b]{2}{*}{ SE } & \multirow[b]{2}{*}{ Z } & \multirow[b]{2}{*}{$p$} & \multirow[b]{2}{*}{$\begin{array}{c}\text { Adjusted } \\
p\end{array}$} & \multirow[b]{2}{*}{ OR } & \multicolumn{2}{|c|}{$\begin{array}{l}\text { 95\% Confidence } \\
\text { Interval }\end{array}$} \\
\hline & & & & & & & & Lower & Upper \\
\hline \multirow{6}{*}{$\begin{array}{l}\text { NEVER able } v s \text {. } \\
\text { ALWAYS able to } \\
\text { access treatment }\end{array}$} & Intercept & -5.37 & 4.27 & -1.26 & 0.22 & 0.44 & 0.00 & $1.07 \mathrm{e}-6$ & 20.04 \\
\hline & Homophobia & -0.02 & 0.05 & -0.07 & 0.94 & 1.00 & 0.97 & 0.49 & 1.93 \\
\hline & Racism & 0.20 & 0.36 & 0.57 & 0.57 & 1.00 & 1.23 & 0.60 & 2.52 \\
\hline & $\begin{array}{l}\text { Income } \\
\text { Inequality } \\
\text { (Gini) }\end{array}$ & -2.05 & 10.02 & -0.20 & 0.84 & 0.84 & 0.13 & $3.7 \mathrm{e}-10$ & $4.4 e+7$ \\
\hline & $\begin{array}{l}\text { Provider } \\
\text { Shortage } \\
\text { (HPSA) }\end{array}$ & 0.42 & 0.12 & 3.44 & $\begin{array}{r}0.00 \\
06\end{array}$ & 0.0012 & 1.53 & 1.19 & 1.94 \\
\hline & $\begin{array}{l}\text { Homophobia * } \\
\text { Racism }\end{array}$ & -0.27 & 0.17 & -1.54 & 0.12 & 0.12 & 0.76 & 0.53 & 1.07 \\
\hline \multirow{6}{*}{$\begin{array}{l}\text { SOMETIMES able } \\
\text { vs. ALWAYS able } \\
\text { to access treatment }\end{array}$} & Intercept & 0.54 & 4.16 & 0.13 & 0.90 & 0.90 & 1.72 & $4.89 \mathrm{e}-4$ & 6023.18 \\
\hline & Homophobia & -0.13 & 0.34 & -0.39 & 0.70 & 1.00 & 0.88 & 0.45 & 1.71 \\
\hline & Racism & -0.09 & 0.36 & -0.25 & 0.80 & 1.00 & 0.91 & 0.5 & 1.86 \\
\hline & $\begin{array}{l}\text { Income } \\
\text { Inequality } \\
\text { (Gini) }\end{array}$ & -9.45 & 9.78 & -0.97 & 0.33 & 0.66 & $7.8 \mathrm{e}-5$ & $3.70 \mathrm{e}-13$ & 16590.93 \\
\hline & $\begin{array}{l}\text { Provider } \\
\text { Shortage } \\
\text { (HPSA) }\end{array}$ & 0.29 & 0.11 & 2.57 & 0.01 & 0.01 & 1.34 & 1.07 & 1.68 \\
\hline & $\begin{array}{l}\text { Homophobia * } \\
\text { Racism }\end{array}$ & -0.49 & 0.21 & -2.30 & 0.02 & 0.04 & 0.61 & 0.40 & 0.93 \\
\hline
\end{tabular}

Note. Reference category for outcome variable: "always" accessing treatment when needed. 


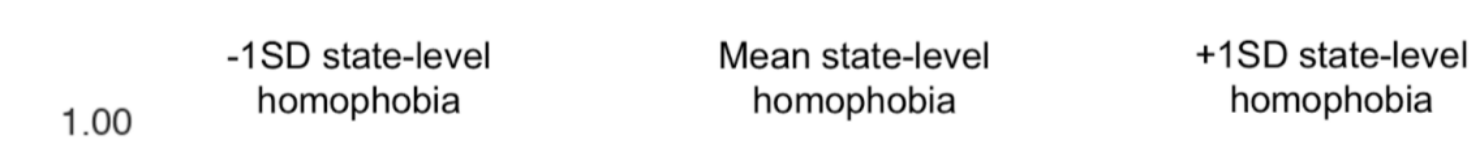

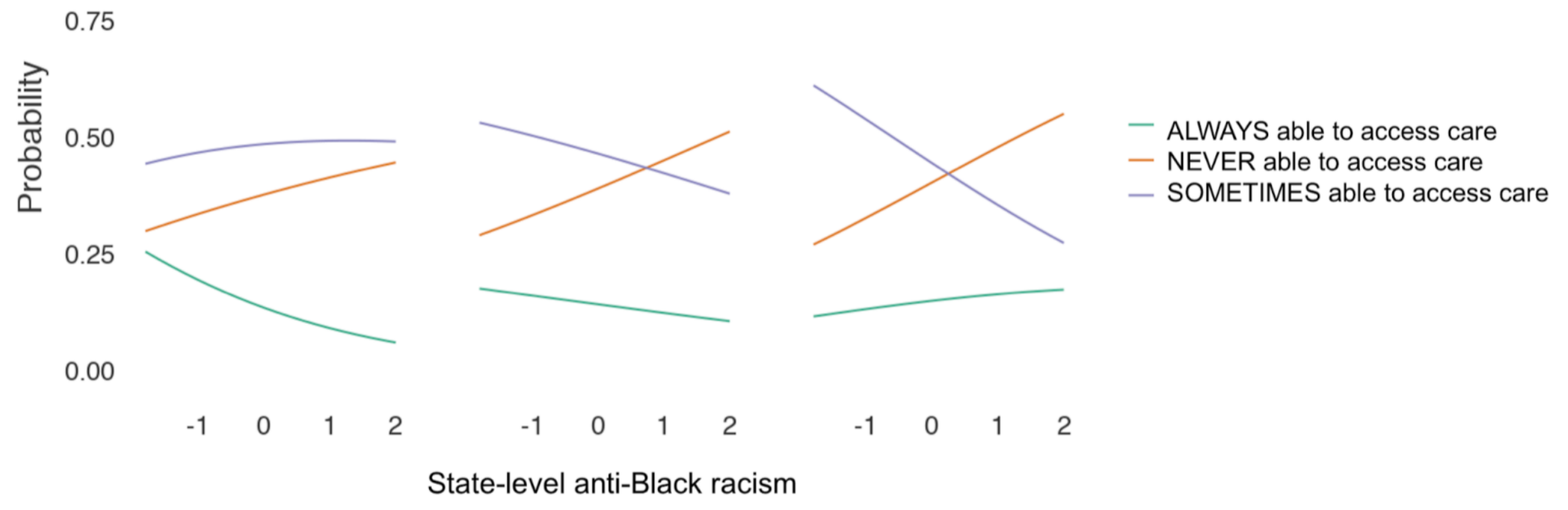

Figure 1. Probability of mental health access during COVID-19 among queer youth of Color living in states characterized by varying levels (-1SD, Mean, +1SD) of anti-Black racism and homophobia. 